\title{
Une carrière mouvementée au Royaume-Uni
}

\section{Peter Satchwell}

Traducteur : Agnès Rambier

\section{OpenEdition \\ Journals}

Édition électronique

URL : http://journals.openedition.org/ries/3384

DOI : $10.4000 /$ ries.3384

ISSN : 2261-4265

\section{Éditeur}

Centre international d'études pédagogiques

\section{Édition imprimée}

Date de publication : 1 mars 1996

Pagination : 41-58

ISSN : 1254-4590

\section{Référence électronique}

Peter Satchwell, « Une carrière mouvementée au Royaume-Uni », Revue internationale d'éducation de Sèvres [En ligne], 09 | 1996, mis en ligne le 20 août 2013, consulté le 23 mars 2021. URL : http:// journals.openedition.org/ries/3384 ; DOI : https://doi.org/10.4000/ries.3384

Ce document a été généré automatiquement le 23 mars 2021.

(c) Tous droits réservés 


\title{
Une carrière mouvementée au Royaume-Uni
}

\author{
Peter Satchwell \\ Traduction : Agnès Rambier
}

1 En écrivant pour une revue internationale, je suis conscient de la nécessité de replacer l'apprentissage précoce des langues étrangères (EFLL) dans le contexte des systèmes éducatifs rencontrés au Royaume-Uni (l'Angleterre, le pays de Galles, l'Irlande du Nord et l'Écosse ont tous des structures bien distinctes, même si elles ont de nombreux points communs). Par conséquent, j'ai divisé cet article en deux sections : la première s'efforce de faire un bref historique de l'EFLL dans les écoles primaires britanniques; la seconde évalue la situation à laquelle nous sommes aujourd'hui parvenus et examine les problèmes majeurs auxquels sont confrontées nos écoles lorsqu'elles projettent de faire figurer les langues étrangères dans le programme scolaire du primaire.

\section{Un peu d'histoire}

\section{Un premier projet en 1960}

L'enseignement des langues aux élèves du primaire en Grande-Bretagne a connu une carrière mouvementée. Il a toujours été synonyme de confusion, et c'est encore le cas aujourd'hui! La première initiative coordonnée a commencé au début des années soixante. Il s'agissait d'un Projet national d'enseignement du français dans le primaire, lequel était cofinancé par le gouvernement et la fondation Nuffield. Une vaste équipe d'experts en linguistique s'est alors rassemblée pour organiser et publier un nouveau cours de français intitulé En Avant, qui proposait un matériel pédagogique destiné aux enfants commençant le français à l'âge de huit ans. Ce cours fut publié en cinq sections, sur une période de dix ans. Les sections un et deux étaient destinées à l'école primaire, les sections trois à cinq s'adressaient aux élèves qui poursuivaient dans le secondaire. À cette époque, la plupart du matériel pédagogique utilisé dans ce cours était révolutionnaire. Ainsi, il insistait beaucoup sur l'utilisation du magnétophone, 
«technologie nouvelle» à l'époque, donnait plus d'importance à un français authentique qu'à des exercices de grammaire artificiels et contenait beaucoup de textes enregistrés dans leur contexte. Les sections destinées au secondaire contenaient beaucoup d'informations culturelles récentes concernant les régions de France, y compris des photos reproduites dans les livres de cours et des films en couleur.

3 En revanche, les auteurs ne surent pas proposer assez d'activités intéressantes destinées à faire s'exprimer les élèves, de sorte que le cours comportait beaucoup d'écoute passive et un trop grand nombre de phrases à répéter mécaniquement («Écoutez et répétez! » disaient constamment les bandes magnétiques!).

4 Ce matériel pédagogique a été largement utilisé au Royaume-Uni (mais en aucune manière par la totalité des écoles) pendant plus de quinze ans avec plus ou moins de succès, en fonction des aptitudes et de l'enthousiasme de l'enseignant. D'un autre côté, de nombreux établissements scolaires du secondaire qui n'étaient pas impliqués dans ce projet pilote continuèrent à enseigner avec des manuels démodés, axés sur la grammaire, qui ennuyaient les enfants et ne leur donnaient aucun aperçu du mode de vie français.

5 Au début des années soixante-dix, la Fondation nationale de recherche pédagogique (NFER) entreprit des recherches sur l'efficacité de l'enseignement du français aux enfants du primaire, et s'efforça de comparer les progrès réalisés par un ensemble d'élèves âgés de treize ans dont certains avaient appris le français dès l'âge de huit ans, et d'autres n'avaient pas commencé avant onze ans, à leur entrée dans le secondaire. Bien que cette enquête fût très poussée, dans la mesure où elle suivait trois groupes d'élèves qui avaient commencé le français à des périodes différentes, elle ne concernait qu'un nombre d'enfants inférieur à 10000 et ses conclusions étaient largement basées sur une série de tests formels.

6 Le rapport de la NFER intitulé Pour ou contre le français dans le primaire ?', est arrivé à la conclusion qu'il n'y avait aucun avantage significatif à commencer le français dès huit ans, et qu'à l'âge de treize ans, ceux qui avaient commencé plus tard, c'est-à-dire à onze ans, avaient quasiment rattrapé ceux qui avaient commencé plus tôt.

7 À la suite de la publication de ce rapport, le financement national et le soutien politique apportés à l'enseignement précoce des langues vivantes (ETML) cessèrent pour ainsi dire du jour au lendemain, et on laissa les écoles libres de choisir si elles voulaient poursuivre cet enseignement ou non. Même si de nombreuses Local Education Authorities (LEAS) abandonnèrent le français au primaire, un petit nombre d'entre elles telles que le Buckinghamshire, le Warwickshire, le Sussex et le Surrey continuèrent à soutenir l'apprentissage précoce des langues tout au long des années soixante-dix et quatrevingt.

8 En revanche, le rapport Burstall a clairement montré que les élèves commençant à apprendre une langue, très jeunes, faisaient preuve d'une attitude positive durable par rapport à l'apprentissage des langues, et d'une très nette supériorité en matière de capacité d'écoute.

9 Mais alors, pourquoi un projet pilote d'une telle ampleur, qui avait coûté au gouvernement plus d'un million de livres a-t-il échoué ? Plusieurs raisons peuvent l'expliquer :

- le manque de personnel qualifié. Personne ne semblait avoir réalisé que plus de la moitié des enseignants prenant part au projet pilote n'avaient qu'une connaissance très limitée du 
français. Ils n'avaient aucune qualification réelle en la matière, et manquaient de confiance et d'aisance ;

- le manque de formation. Aucun des enseignants concernés n'avait reçu de formation spécifique, concernant la langue elle-même, ou la méthodologie à employer avec ce groupe d'âge, avant de commencer à enseigner avec un matériel nouveau qu'ils ne connaissaient pas $\mathrm{du}$ tout. De plus, c'était la première fois au Royaume-Uni que les écoles essayaient d'enseigner une langue étrangère à tous les niveaux ;

- le manque d'organisation. Il n'existait aucun accord au niveau national concernant le nombre d'heures qui devrait être dévolu au français, de sorte qu'il n'y avait aucune uniformité entre les différentes écoles ;

- le manque de financement. Peu d'écoles ont reçu la somme d'argent supplémentaire nécessaire à l'achat du cours Nuffield. La plupart des subventions gouvernementales étaient allées au développement des matériels, mais pas aux écoles elles-mêmes.

10 De la sorte, l'apprentissage précoce des langues disparut des priorités nationales pendant presque vingt ans. Cependant, depuis 1989 se sont produits certains événements qui ont concouru à faire renaître l'intérêt des parents, des enseignants et, petit à petit, des industriels.

\section{Les années quatre-vingt-dix}

11 L'Education Act de 1989 fit naître pour la première fois en Angleterre et au pays de Galles l'idée d'un programme national unifié et centralisé pour toutes les écoles du primaire et du secondaire (à l'exception des écoles privées !). Ceci avait pour fonction de garantir à tous les élèves un programme uniforme et équilibré comprenant l'enseignement des mathématiques, de l'anglais, des sciences, de l'histoire, de la géographie, des arts plastiques et de la technologie tout au long de leur scolarité, c'est-à-dire de cinq à seize ans. Pour la première fois également, tous les enfants étaient obligés d'apprendre une langue étrangère (une seulement!) entre onze et seize ans. Ironie du sort, la langue étrangère est la seule matière à ne pas figurer dans le cycle primaire.

Depuis 1990, de nombreux parents ont commencé à faire pression sur les écoles primaires pour qu'elles commencent à enseigner une langue étrangère, parfois dès l'âge de cinq ans. Lorsque les directeurs d'établissement ne disposent pas de personnel enseignant ayant les compétences linguistiques nécessaires, ils mettent souvent en place des clubs de langues fonctionnant après les heures de cours ou le samedi matin et pour lesquels les parents doivent payer des frais de scolarité. (Il est peut être significatif qu'un organisme privé appelé «Club français » soit, en cinq ans, passé du stade de petite industrie artisanale à celui d'important réseau national d'enseignants franchisés qui animent des clubs d'enfants âgés de quatre à dix ans et dont le chiffre d'affaires annuel est de 500000 livres !).

Depuis 1989, le gouvernement a débloqué des crédits substantiels pour le financement d'un important projet pilote mené en Écosse par le Scottish Office Education Department $\left(\mathrm{SOED}^{2}\right)$.

Ce projet a remporté un tel succès qu'il a attiré l'attention internationale. Je vais donc constamment m'y référer dans cet article. Ce projet pilote de cinq ans s'est terminé en 1994, mais son succès a été tel que le gouvernement a dépensé plusieurs millions de livres pour financer un important programme national de recyclage qui va se dérouler au cours des trois prochaines années. Plusieurs universités écossaises apportent leur 
concours à ce programme dont le but est d'offrir vingt-sept journées (cent soixante heures) de formation linguistique (français, allemand, italien et espagnol) et méthodologique à plus de deux mille cinq cents enseignants du primaire, un pour chaque école primaire écossaise, dans le but de les préparer à enseigner à deux niveaux d'élèves minimum, c'est-à-dire aux élèves de dix et onze ans qui sont en P6 et P7 (les élèves écossais passent dans le secondaire à l'âge de douze ans).

Parallèlement à ce projet écossais, les cinq dernières années ont aussi vu naître de nouvelles initiatives locales financées par les LEA et par les écoles en Angleterre et en Irlande du Nord. Les plus importantes ont eu lieu dans l'East Sussex, le West Sussex, le Kent, le Surrey, le Richmond, le Hampshire, le North Yorkshire et tout dernièrement, le Gloucestershire ${ }^{3}$.

16 Étant donné que, de toute évidence, le gouvernement ne va pas prochainement financer l'enseignement précoce des langues dans les écoles primaires d'Angleterre, du pays de Galles et d'Irlande du Nord, les écoles en sont réduites à se débrouiller toutes seules. Dans ce contexte, le Centre d'information sur la recherche et l'enseignement des langues (CILT), situé à Londres, a récemment décidé d'organiser des conférences nationales destinées aux enseignants du primaire qui enseignent, ou prévoient d'enseigner les langues étrangères à leurs élèves. Le CILT a également lancé une nouvelle collection, Young Pathfinders destinée à aider et encourager les enseignants du primaire qui ne sont pas nécessairement des spécialistes en langues.

\section{Des questions-clés}

Quelles sont donc les principales questions auxquelles sont confrontées nos écoles primaires lorsqu'elles prévoient de faire figurer une langue étrangère au programme? En bref, elles doivent répondre aux questions suivantes :

Pourquoi ? Qui ? Quelle langue? Quoi ? Quand ? Avec quelle fréquence ? Pour combien de temps? Dans quel but?

\section{Pourquoi ?}

19 Je pense que chaque école doit être très claire sur les raisons pour lesquelles elle veut inclure une langue étrangère dans son programme. Qu'est-ce que l'apprentissage d'une langue étrangère va apporter à la culture générale des enfants et comment va-t-il s'inclure au programme suivi par l'école?

20 Voici quelques-unes de nos préoccupations principales en Grande- Bretagne: combattre le sentiment d'insularité chez les parents comme chez les enfants, et ouvrir les yeux et les oreilles des enfants à d'autres cultures. En Grande-Bretagne, nous ne sommes pas constamment exposés aux langues étrangères dans les médias, comme c'est souvent le cas dans le reste de l'Europe: dans presque toutes les émissions, les hommes politiques, les personnalités sportives et même les pop stars voient leurs paroles immédiatement couvertes par la voix du traducteur, qui se fait entendre dès l'instant qu'ils ouvrent la bouche pour s'exprimer dans une langue étrangère !

21 Les Britanniques ont, au sein de l'Europe industrielle et commerciale, la mauvaise réputation, bien méritée, d'être des linguistes incompétents et paresseux. C'est là une triste réalité historique, mais des générations entières ont quitté l'école, jusqu'en 1988, 
avec une expérience d'apprentissage des langues très négative, aboutissant à l'obtention d'une mauvaise note d'examen à l'âge de seize ans. Ceci est en train de changer du fait de meilleures méthodes d'enseignement et d'examens plus appropriés, mais les écoles britanniques sont toujours largement à la traine par rapport à la plupart des autres pays d'Europe, puisqu'elles ne proposent que deux cours de langue par semaine (cent vingt cent quarante minutes maximum), et ceci pendant cinq ans seulement, dans les écoles du secondaire, ce qui ne représente qu'un total de trois cent soixante heures.

Ceci signifie que la plupart des élèves anglais passent moitié moins de temps que leurs homologues allemands ou hollandais à apprendre des langues étrangères. Voilà donc encore une bonne raison de commencer plus tôt.

Le seul avantage réel à faire commencer les élèves tôt réside dans le fait que cela permet de gagner l'intérêt et l'enthousiasme des enfants avant le début de la puberté et le comportement timide et gauche qui l'accompagne. Les enfants du primaire absorbent une langue nouvelle aisément et sans inhibition.

Il est aussi très clair que l'enseignement précoce des langues étrangères augmente la confiance en soi et développe la conscience du langage en général, ce qui renforce et enrichit la connaissance de sa propre langue.

Il existe bien entendu beaucoup d'autres raisons pour commencer à apprendre une langue de bonne heure, qui sont spécifiques à chaque pays et à sa culture.

\section{Qui ?}

Le plus gros problème auquel sont confrontées les écoles est le suivant: qui va enseigner la langue étrangère?

Nous avons tout essayé en Angleterre au cours de ces quinze dernières années :

- le linguiste «spécialiste » qui possède un diplôme universitaire et fait le tour de plusieurs écoles primaires pour faire au mieux deux cours de trente à quarante minutes par semaine dans chaque école ;

- le locuteur natif qui parle bien sûr couramment, mais qui peut n'avoir reçu aucune formation ou une formation insuffisante pour enseigner à des enfants du primaire ;

- l'enseignant qui n'a aucune qualification officielle, mais qui a des contacts réguliers avec la France et que l'enseignement du français enthousiasme ;

- l'enseignant qui n'a aucune qualification, mais que l'on a persuadé de faire le cours de français parce que Madame Untel est partie, etc.

Nous avons aussi un problème constant en Grande-Bretagne, qui provient du fait que nombre de professeurs de langues du secondaire font preuve d'une attitude très arrogante envers leurs collègues du primaire qui essayent d'enseigner une langue. Cette arrogance est déplacée et doit disparaître, car je pense réellement que la plupart des enseignants du secondaire pourraient beaucoup apprendre en matière de pratique pédagogique et de gestion de classe en assistant aux cours d'un collègue du primaire pendant une semaine! Ce qui inquiète évidemment les linguistes du secondaire, c'est que l'enseignant du primaire fasse des erreurs dans la langue d'arrivée et enseigne aux enfants un mauvais français, un mauvais allemand ou espagnol.

Mais ils oublient que c'est ce qu'ils font eux-mêmes chaque semaine! Alors, comment définir l'enseignant idéal de langues dans le primaire ? Est-il réellement nécessaire qu'il 
possède intégralement la connaissance théorique d'une langue, celle que l'on attend d'un diplômé de l'université qui a passé quatre ou cinq ans à étudier des ouvrages ampoulés de littérature et de linguistique?

Au Royaume-Uni, nous venons juste de commencer à définir les savoirs et les savoirfaire dont un enseignant du primaire a vraiment besoin pour offrir à ses élèves un enseignement valable et gratifiant.

31 Un de nos maîtres assistants, spécialisé dans la formation des enseignants du primaire non spécialistes a récemment proposé quatre priorités pour l'enseignement des langues dans les écoles primaires, les quatre « $C »$ :

- la communication. On apprend avant tout une langue pour communiquer, c'est pourquoi on devrait insister dans les écoles primaires sur l'écoute et la prise de parole; et la communication doit se faire "pour de vrai», ce qui veut dire que l'enseignant devrait utiliser constamment la langue d'arrivée dans la vie quotidienne de la classe, organiser des activités et des jeux. La classe doit dégager une certaine atmosphère, l'ambiance réelle des pays dont la langue est étudiée, et contenir de nombreux objets provenant des pays en question que les enfants peuvent voir et toucher;

- la culture. Il est impossible d'enseigner une langue en-dehors de tout contexte culturel. Elle doit être enseignée en même temps que la culture dans laquelle elle s'inscrit. Ainsi, les enfants doivent apprendre les mœurs, les usages et les coutumes du pays pour parler la langue correctement et la ressentir. Ils doivent en quelque sorte s'identifier aux personnes qui parlent la langue qu'ils apprennent et apprendre à faire la différence entre la culture authentique du pays et les stéréotypes nombreux et dangereux perpétués par les médias, l'industrie touristique et leurs propres parents;

- le contexte. La langue doit être enseignée dans un contexte réel qui ait une signification pour l'enfant. Cela veut dire que l'enseignant doit soigneusement choisir des thèmes qui intéressent et motivent la classe parce qu'ils sont directement liés à la vie des élèves. Après avoir présenté une langue étrangère, l'enseignant doit encourager les enfants à s'approprier cette langue, en leur donnant de nombreuses occasions de l'utiliser et de la mettre en scène pour ainsi dire, en créant leurs propres dialogues, courtes pièces de théâtre, histoires, poèmes, comptines, raps et chansons.

- la confiance. Il est crucial d'aider les élèves à avoir confiance en eux lorsqu'ils parlent une langue étrangère. Ils ont besoin d'aimer ce qu'ils apprennent et de "savoir ce qu'ils savent ", afin de pouvoir utiliser correctement les structures qu'ils ont apprises. Cela implique du doigté de la part de l'enseignant lorsque l'élève fait des erreurs. Aucun enfant n'aura envie de parler si on le corrige constamment et si on lui dit qu'il se trompe. Les enseignants doivent eux aussi avoir confiance en eux-mêmes! Ils doivent apprendre à accepter leurs propres erreurs et à les corriger avec l'aide des élèves. Des méthodes pédagogiques de qualité sont plus importantes que la compétence linguistique en elle-même.

«En pratique, le français primaire prime sur le français!» Un bon enseignement des langues dans le primaire devrait aider les enfants à se sentir à l'aise par rapport au langage en général et à la langue étrangère qu'ils étudient en particulier. Il devrait éveiller l'enfant au langage en tant que phénomène humain tout en développant chez lui une attitude positive par rapport à l'apprentissage des langues ${ }^{4}$.

L'important programme de formation actuellement coordonné en Écosse par le Scottish Office Education Department (SOED) a permis de définir de façon encore plus détaillée les compétences nécessaires à l'enseignant du primaire : cette formation a non seulement pour fonction d'enseigner la langue parlée dans la classe, mais aussi celle qu'il faut 
connaître lorsque l'on est en contact avec des locuteurs natifs chez soi ou à l'étranger. À cet égard, cette formation est proche des ouvrages de formation récemment écrits par Renée Birks, de l'université de Glasgow, intitulés Une vie d'Instit et Travail d'Instit ${ }^{5}$.

Selon le programme de formation du SOED, l'enseignant du primaire devrait apprendre à maîtriser :

- le système qui régit la langue, une prononciation et une intonation de qualité ;

- l'alphabet et les nombres ;

- le vocabulaire de base dans les domaines suivants : le vocabulaire que l'on utilise pour parler de soi, de sa famille, de l'endroit où l'on habite ; le vocabulaire qui sert à décrire les gens, les animaux, les vêtements, les maisons, la ville, l'environnement, le temps, la nourriture et la boisson; le vocabulaire affectif qui sert à exprimer ce que l'on aime ou non, ses sentiments, ses émotions, ses douleurs, ses compliments, son affection;

- le vocabulaire de classe : les activités quotidiennes; la langue utilisée par l'enseignant pour saluer les élèves, leur donner des instructions, etc.; la langue utilisée par l'enseignant pour organiser les activités des élèves; la langue utilisée par les élèves pour demander la permission de faire quelque chose, expliquer leurs problèmes, etc.

- la langue nécessaire pour transmettre des notions de base en matière d'art, de travaux manuels, d'économie domestique, de science, de technologie, de théâtre et d'activités physiques : dessiner/couleur/mesurer/couper/plier/coller, etc., les activités saisonnières, les parties du corps ; courir/sauter/soulever/attraper/être debout/être allongé, etc.

- la langue nécessaire pour jouer ; pour apprendre aux élèves des poèmes, des comptines et des chansons; pour raconter et mimer, en les enrichissant, des histoires populaires au moyen de la langue étrangère.

Tous les domaines linguistiques énoncés ci-dessus ne peuvent être correctement enseignés que si l'enseignant possède bien la structure de base de la langue et ses modes de fonctionnement. Le programme de formation écossais insiste par conséquent sur la nécessité d'étayer les compétences grammaticales :

«L'enseignant du primaire devra posséder les structures de base de la langue, être capable d'utiliser correctement un dictionnaire de langue étrangère et de libeller un texte court à l'aide de celui-ci. Il devra acquérir des connaissances concernant la culture et le cadre de vie des pays dont la langue est enseignée ${ }^{6} »$.

Il semble logique, si l'on veut que les enfants du primaire aient une première expérience positive et gratifiante, que leurs enseignants soient correctement formés à la méthodologie qui convient à ce type d'élèves. Ils doivent être suffisamment compétents et à l'aise dans la langue étrangère parlée dans une classe de primaire. Il ne s'agit en aucune façon de "délayer" ou d'adapter les techniques et le matériel pédagogiques destinés aux élèves du secondaire! La question essentielle réside, sans aucun doute, dans la nature et le coût d'une formation adéquate, et tous ceux d'entre nous qui sont prêts à se battre pour que la même formation ait lieu en Angleterre, au pays de Galles et en Irlande vont surveiller ce projet écossais de très près afin de tirer d'éventuelles leçons de ce travail totalement nouveau.

\section{Quelle langue?}

Le choix de la langue enseignée est, dans la plupart des écoles, fonction des enseignants disponibles et de leurs connaissances. Malheureusement, au Royaume-Uni cela signifie que $90 \%$ des écoles enseignent le français, car c'est la langue que la plupart des 
enseignants ont apprise à l'école. Cela ne correspond pas forcément aux vœux de tous les parents et ce n'est certes pas ce dont le pays a besoin à l'aube du XXI siècle. Cependant, comme il ne reste que quatre instituts de formation des maîtres qui offrent aux stagiaires du primaire l'option d'une langue étrangère pendant leurs quatre ans de formation, la perspective d'accroître le nombre d'enseignants compétents en langues demeure sombre. Les Écossais, eux, ont pris soin de diversifier les langues enseignées dans le cadre du projet pilote, de sorte que l'espagnol, l'italien, et l'allemand ont aussi été inclus à la formation.

Certains parents vont inévitablement faire pression pour que les élèves passant dans le secondaire continuent la même langue. De nombreux directeurs d'écoles primaires auront sans doute à résister à de telles pressions, c'est pourquoi nous devons réfléchir avec le plus grand soin à la nature et à l'objectif de l'enseignement d'une langue étrangère dans le primaire. Ceci nous conduit au débat suivant: l'expérience du primaire doit-elle être considérée comme une sorte d'«apprentissage» destiné à apprendre comment apprendre une langue, n'importe quelle langue, ou doit-elle être envisagée comme un processus continu d'apprentissage de la même langue, susceptible de commencer à l'âge de cinq ans et de finir à l'âge de seize ans! Si l'on opte pour le premier cas, la langue choisie à l'école primaire a peu d'importance, puisque le cours peut être perçu comme un tout indépendant. En optant pour le deuxième cas, on s'expose aux innombrables questions qui se posent actuellement en France comme en Angleterre. Comment peut-on raisonnablement assurer une continuité entre le primaire et le secondaire lorsque, au sein d'une douzaine d'écoles primaires ou davantage qui envoient leurs élèves dans un établissement secondaire, il n'existe même pas d'uniformité quant à l'enseignement prodigué ? Je pense que nous devrions adopter une attitude beaucoup plus souple face à ce problème. Si nous suivons les sages conseils qu'Eric Hawkins donnait dès 1981 dans son livre intitulé Les Langues vivantes au programme, nous devrions prévoir une phase d'apprentissage où les enfants du primaire sont encouragés à explorer plusieurs langues dans le cadre d'un programme bien structuré de "prise de conscience du langage ", qui se déroule simultanément à l'apprentissage de leur première langue étrangère ou bien avant cet apprentissage. Eric Hawkins proposait un programme tout à fait passionnant pour le secondaire, mais la plupart des activités proposées ne conviendraient pas du tout à des enfants en P 4, 5 ou $6^{7}$.

\section{Quoi?}

En tentant de définir quels domaines linguistiques et culturels conviennent aux enfants du primaire, on semble parvenir à une sorte de consensus international qui tend vers un petit nombre de thèmes-clés, pouvant être déclinés à l'infini, selon l'âge et les centres d'intérêt des élèves. On semble toujours commencer par :

- « moi » : ma famille, mon corps, mes objets favoris, ce que j'aime et ce que je n'aime pas ;

- « ma maison » : ma maison, mes animaux, mes amis ;

- « ma ville / mon village » : l'endroit où j'habite, les alentours ;

- « mon école » : préférences et activités ; et à partir de là, on encourage les enfants à regarder autour d'eux, à s'intéresser à des thèmes qui les impliquent progressivement au «monde extérieur » : l'environnement, la nature, la protection de l'environnement, comment la nourriture est produite, comment les choses sont fabriquées... 
40 pensé à faire plus de trois ou quatre cours de langues discontinus par semaine. De nombreux enseignants prenant part au projet pilote ont exploré les possibilités d'« enchâsser " l'apprentissage de la langue étrangère dans la totalité du programme scolaire, afin que les enfants perçoivent cette langue comme un outil concret de communication, dont ils ont constamment besoin lors de cours habituels, quel qu'en soit le sujet. Par exemple, le calcul mental est régulièrement pratiqué en allemand, les cours d'éducation physique sont donnés en espagnol, les recettes de cuisine sont données en français, les enquêtes de classe (par exemple : qui aime tel ou tel parfum de glace ?) et les relevés des résultats sont faits en italien.

Cette pratique ne signifie pas une « immersion partielle » pour les Écossais, du type de celle des modèles canadien et australien désormais célèbres. Les concepts principaux de chaque thème continuent, dans la plupart des cas, à être enseignés dans la langue maternelle (L1), mais à chaque fois que cela est possible, des parties de cours ou des cours entiers viennent renforcer le travail de la langue 2. Ce qui signifie que les enseignants écossais ont découvert de nombreuses façons d'utiliser la langue 2 dans des activités de classe quotidiennes, telles que faire l'appel, parler du temps, de ce qu'on a fait pendant le week-end, des activités qu'on va pratiquer dans la journée, tout ceci permettant à la langue étrangère de se manifester beaucoup plus qu'au cours de seulement trois sessions hebdomadaires d'une demi-heure.

Bien sûr, enseigner une langue étrangère signifie aussi enseigner la culture des pays dans lesquels elle est parlée. Les enfants ont besoin de connaître des chansons, des comptines ou des histoires du pays étranger. Beaucoup de contes de fée et de fables sont internationaux, mais les enfants apprennent volontiers et mettent en scène leur propre version d'une histoire bien racontée en réalisant des costumes, des masques et des supports. Des enfants écossais ont été filmés en train de jouer l'histoire du petit éléphant qui voulait avoir un long nez, Le Petit Chaperon rouge et beaucoup d'autres histoires, qui impliquaient souvent une participation physique totale, ou en train de mimer des chansons. La Chasse à l'ours est populaire dans n'importe quelle langue et à tout âge, de même que les jeux où on compte et où on saute à la corde, qui sont appréciés dans la cour de récréation. Un nombre varié de jeux pratiqués dans les cours de récréation françaises est présenté dans les excellentes cassettes pour Le petit Trampoline $e^{8}$.

43 Les enfants sont toujours curieux de savoir comment les enfants de leur âge vivent dans d'autres pays, et les écoles primaires devraient essayer de créer des liens avec des écoles à l'étranger, échanger des lettres, des cassettes, des colis mystérieux, du matériel pédagogique, des photos et des vidéos de l'école et de la ville ou du village, de la vie quotidienne des gens qui vivent là-bas. Bien sûr, l'idéal serait que tous les enfants du primaire en Europe fassent leur premier voyage à l'étranger avant l'âge de dix ans, et de nombreuses écoles primaires ont aujourd'hui compris combien il est profitable d'emmener leurs élèves une semaine à l'étranger, en dépit des problèmes financiers que cela représente dans de nombreuses régions.

On devrait inculquer aux enfants que leur culture n'est pas la seule valable et qu'ils vivent dans un monde multiculturel et multilingue complexe, qu'ils soient en Angleterre, en Écosse, en France, en Espagne ou en Allemagne. C'est pourquoi il est important de leur faire partager l'excitation des fêtes nationales et religieuses célébrées dans le pays étranger - Noël, le Nouvel An, Pâques, les anniversaires, les 
fêtes - car tous ces thèmes doivent impliquer les élèves dans des activités manuelles (faire des cartes de vœux, des recettes, des œufs de Pâques décorés, etc.) dont le résultat peut être ultérieurement exposé dans l'école.

\section{Comment ?}

45 Avant toute chose, l'apprentissage d'une langue doit être amusant. Les enfants doivent prendre plaisir à pratiquer les activités du cours de langue, et l'enseignant doit s'amuser tout autant que les enfants. Par conséquent, l'enseignant peut avoir à redéfinir son rôle, à s'éloigner de l'approche didactique traditionnelle pour devenir tour à tour celui qui présente une langue nouvelle, l'animateur, celui qui aide les enfants, l'ami des enfants, l'oreille indiscrète!

Les cours doivent être organisés de façon à garantir une utilisation maximale de la langue d'arrivée, à travers de nombreuses activités et l'implication des élèves qui utilisent la langue pour parler de ce qui les touche. Les cours doivent aussi offrir aux élèves de multiples occasions de pratiquer la langue pendant que l'enseignant écoute. Il est également important d'offrir aux élèves un choix varié d'activités, afin qu'ils puissent apprendre à leur propre rythme.

Nous devons aussi nous assurer que les cours de langues ne deviennent jamais fastidieux, ni ennuyeux, comme c'était trop souvent le cas par le passé! Cela implique d'utiliser toute une panoplie de techniques et de matériels pédagogiques, qui comprenne un répertoire multimédia de cassettes audio et vidéo, des transparents pour rétroprojecteurs, des supports visuels pour la classe et pour le pair work, des logiciels et tout un ensemble de documents visuels ${ }^{9}$ et écrits.

48 Afin d'explorer les infinies possibilités des activités destinées à pratiquer une langue étrangère en classe de primaire, je ne saurais que trop recommander aux lecteurs de se plonger dans les dix numéros du magazine PRIMAR ${ }^{10}$. Ils peuvent aussi prendre connaissance d'extraits de cours extrêmement variés et tous excellents; il s'agit d'extraits de cours de français, d'allemand, d'italien et d'espagnol enregistrés en 1993 dans des écoles primaires écossaises ${ }^{11}$.

\section{Dans quel but?}

Les écoles primaires doivent organiser l'enseignement d'une langue étrangère avec autant de soin qu'une toute autre partie du programme. Les enseignants doivent avant tout prévoir une progression en cours d'année et fixer, tant pour eux-mêmes que pour leurs élèves, des objectifs à court terme facilement évaluables. Ces objectifs ne doivent pas être des tests formels. Ils doivent conduire les élèves à exécuter des tâches amusantes, telles qu'interpréter un court dialogue ou une simple saynète, en les encourageant à se déguiser et à utiliser des supports, à improviser le plus souvent possible.

Une autre approche, qui combine les aspects culturels et linguistiques, définit un ensemble d'environ trois à cinq grands thèmes sur l'année, de façon à ce que les élèves ne soient pas précipités d'activités en activités qui n'ont pas de rapport entre elles. Trampoline 1 est un bon exemple de cette approche. Le matériel y est présenté sous la forme de cinq magazines, qui permettent aux enfants de suivre une année scolaire dans 
une école primaire française, en les emmenant de la «rentrée » aux «grandes vacances » sur trois trimestres.

Enfin, nous devons nous assurer que la progression des savoir-faire de l'apprenant soit bien prise en compte. Nous espérons que, tout au long de l'année, ils auront acquis un certain nombre de savoir-faire principaux, et nous devrions définir et décrire ces derniers. Par exemple, être capable de compter, de pratiquer des activités artistiques (modelage, dessin, musique), corporelles (jeux de balle, mouvements). Ces savoir-faire doivent ou devraient faire partie des cours de langues à un moment ou à un autre de l'année.

En conclusion, je proposerai que nos buts et nos objectifs principaux lorsque nous enseignons des langues à de jeunes apprenants recouvrent les points suivants :

- développement des capacités communicatives et linguistiques. Développer chez l'enfant la conscience et la compréhension de base du processus d'apprentissage d'une langue nouvelle ; développer chez chaque enfant la capacité à communiquer dans la L2 à un niveau approprié ; permettre à tous les enfants d'utiliser la langue de façon créative et efficace, et en tirer satisfaction ;

- développement des savoir-apprendre. Développer la capacité à écouter attentivement; développer la confiance en soi et la capacité à parler devant différents types de publics, y compris des locuteurs natifs; apprendre à apprendre de façon générale et développer la capacité à trouver des informations par soi-même; apprendre à bien se servir d'un ordinateur ;

- développement de la conscience sociale et culturelle. Encourager les enfants à travailler ensemble, à coopérer; développer, chez l'enfant, un intérêt pour le pays étranger et une attitude positive envers ses habitants et ses coutumes; développer chez l'enfant l'enthousiasme à apprendre une langue étrangère; créer un environnement dans lequel chaque enfant, quelles que soient ses capacités, puisse connaître la réussite et acquérir de plus de confiance en lui, en utilisant la langue étrangère dans des situations réelles.

Je crois que nous avons encore énormément de recherches à faire pour comprendre comment les enfants acquièrent des langues et comment nous devrions les leur enseigner. Nous devrions davantage mettre en commun, et de façon plus régulière, toutes les bonnes idées et les expériences réussies qui se rencontrent actuellement dans de nombreuses écoles primaires d'Europe. Nous avons tous beaucoup à apprendre de nos collègues de Hongrie, de Slovénie, d'Autriche, d'Allemagne, d'Italie, de Lituanie, de Pologne, de Russie et de Bulgarie quant à ce qui peut être réalisé dans une classe du primaire. Je ne peux que conclure en émettant le vœu que, dans un avenir proche, tous les élèves du primaire connaîtront, dans leurs cours de langues, de l'enthousiasme et du plaisir à utiliser une langue nouvelle ; une curiosité accrue par rapport au langage en général et à son mode d'utilisation; de l'intérêt pour les gens qui parlent la L2, et les pays où elle est parlée; le plaisir intense d'utiliser la L2 à ses propres fins et d'être compris par des locuteurs natifs; l'acquisition d'une meilleure oreille et une réelle amélioration de leurs capacités d'écoute; le plaisir de pratiquer un nombre très varié de tâches et de jeux, qui les amènent à parler constamment la L2 en classe ; une base solide de compréhension, d'enthousiasme et de confiance en eux-mêmes qui leur permette d'aborder d'autres langues étrangères dans le secondaire ou plus tard à l'âge adulte. 


\section{BIBLIOGRAPHIE}

SOED, Guidelines for Teachers National Pilot: Modern Languages in the Primary School, 1990 et 1992.

SOED, Foreign Languages in the Primary School: the National Pilot Project in Scotland, Interchange, $n^{\circ} 19,1993$.

SOED, Foreign Languages in the Primary School: Competences, 1994. SCOPE et SOED, Modern Languages in the Primary School: Three Videos and Viewing Guide, 1994; disponible chez SCOPE Picture Productions Ltd., Keppie House, 147 Blythswood Street, Glasgow, G2 4EN.

Hawkins Eric, Modern Languages in the Curriculum, Cambridge University Press, 1981 et 1997. Hawkins Eric, Awareness of Language: An Introduction, Cambridge University Press, 1984. Johnstone Richard, Teaching Modern Languages at Primary School Approaches and Implications, The Scottish Council for Reasearch in Education (SCRE), 1994.

Sharpe Keith, The Four Cs of Primary Modern Language Teaching, Language Learning Journal, $\mathrm{n}^{\circ} 6$, 9/1992, published by the Association for Language Learning (ALL).

Young Pathfinder Series, published by the Center for Information on Language Teaching and Research (CILT, 20 Bedfordbury, London, WC2N 4LB), 1995:

Satchwell Peter, De Silva June, YPFI Catching Them Young.

Martin Cynthia, YPF2 Games and Fun Activities.

Dobson Pat et Tierney Danny, YPF3 Story telling.

\section{ANNEXES}

\section{Document 1}

\section{Le projet pilote d'enseignement primaire en Écosse 1989-1994}

1989 : création de six projets pilotes : chaque projet regroupe une école secondaire et la totalité de ses partenaires du primaire. 1990 : adjonction de six autres projets pilotes, ce qui représente un total de douze écoles secondaires et de soixante-seize écoles primaires. Six projets concernent l'enseignement du français, quatre celui de l'allemand, un celui de l'italien et un celui de l'espagnol. Le projet ne commence qu'avec les classes de P7 (enfants de dix-onze ans). Le SOED paie un professeur supplémentaire par projet, qui enseigne à la fois dans le primaire et le secondaire. Les enseignants du secondaire ainsi libérés de leurs classes peuvent aller visiter le groupe d'écoles primaires associé au projet. Dès le début, les enseignants du primaire se sentent très impliqués et sont les premiers à intégrer la langue étrangère dans le programme du primaire.

De nombreuses classes du primaire ont des classes composites, constituées de plus d'un groupe d'âge ; de ce fait, les enfants sont impliqués dans l'apprentissage d'une langue étrangère dès l'âge de sept-huit ans.

1991 : Le SOED étend le projet aux élèves de P6 (enfants de neuf-dix ans). 1992 : deux des projets originaux sont étendus aux P4 (enfants de sept-huit ans). 
Les caractéristiques du projet

Les enseignants du primaire et du secondaire sont délibérément regroupés, afin que les enseignants du secondaire qui possèdent la connaissance de la langue découvrent les méthodes d'enseignement utilisées au primaire, et que les enseignants du primaire apprennent eux aussi les méthodes d'enseignement d'une langue étrangère et acquièrent davantage d'aisance en travaillant aux côtés du linguiste du secondaire et en pratiquant quotidiennement la langue avec les enfants. Ceci ne peut que favoriser la continuité et la coopération entre primaire et secondaire dans les années futures. Aucun manuel n'est utilisé. Les enseignants choisissent les thèmes abordés en fonction des centres d'intérêt des élèves.

Le français, l'allemand, l'italien et l'espagnol sont introduits dans diverses régions d'Écosse. Les enseignants sont encouragés à expérimenter et à explorer toutes les façons d'intégrer la langue étrangère aux activités quotidiennes et au travail poursuivi par la classe. Ainsi la langue étrangère est-elle utilisée pour mimer, chanter, faire du théâtre, raconter une histoire, faire des mathématiques, des activités artistiques, des études environnementales et de l'éducation physique.

Les enseignants bénéficient d'un soutien constant de la part de conseillers et d'enseignants consultants. Le linguiste du secondaire formé pour visiter les classes primaires n'existe plus. On préfère désormais former l'enseignant du primaire luimême.

À l'avenir, l'enseignement de la langue étrangère dans les écoles primaires sera assuré par un enseignant du primaire qui a suivi une année de formation. C'est ce qu'on appelle le principe du drop in ${ }^{12}$ puisque l'enseignant aura à enseigner la langue étrangère aux classes de ses collègues en plus de la sienne.

\section{Document 2}

La LEA de l'East Sussex finance l'enseignement du français en classe de P6 dans toutes ses écoles primaires depuis 1980. Elle utilise des enseignants qui exercent sur plusieurs établissements de même que des professeurs principaux. Une méthode d'enseignement intitulée Salut ! a été publiée en 1981.

La LEA du Kent a, pendant cinq années, procédé au recyclage de plus de cent cinquante enseignants du primaire, afin de leur permettre d'enseigner le français (ou l'espagnol, le portugais ou l'allemand) aux classes de P6 dans environ la moitié de ses écoles primaires. Ces enseignants reçoivent le soutien d'un enseignant consultant et bénéficient d'un matériel pédagogique de grande qualité, Pilote, publié en 1994 sous la forme de trois cassettes vidéo.

La LEA du Surrey a publié un certain nombre de propositions concernant l'EPLV en 1993, qu'elle a fait suivre d'un cours de recyclage pour quinze professeurs principaux entre 1993 et 1995.

La LEA du London Borough of Richmond a financé le recyclage d'un enseignant pour chacune de ses vingt-cinq écoles primaires, afin qu'ils puissent enseigner le français à des élèves de P6. La LEA a également publié une méthode d'enseignement intitulée : C'est facile comme bonjour ! Les enseignants reçoivent le soutien d'un enseignant consultant. A Basingstoke, dans le Hampshire, un projet financé par des industriels recrute des assistants étrangers venant d'Espagne, de France et d'Allemagne dans 
certaines de ses écoles primaires. L'assistant entreprend des activités d'ordre pratique (cuisine, modelage, éducation physique ou jeux) avec les enfants dans le cadre de la classe, sous la surveillance du professeur principal.

Dans le West Sussex, un projet pilote récent d'une $L E A$, qui concerne quatre écoles primaires, est en train d'expérimenter l'enseignement simultané de deux langues étrangères (le français et l'allemand) à des élèves de $\mathrm{P} 6$ et $\mathrm{P} 7$. Jusqu'ici, les réactions des élèves, des enseignants et des parents ont été très positives.

Dans la LEA du North Yorkshire, un groupe d'écoles primaires a commencé à enseigner l'allemand avec le soutien d'une entreprise commerciale et de la LEA. Depuis 1995, la LEA du Gloucestershire encourage toutes ses écoles primaires à envisager l'introduction d'une langue (le français principalement) avec le soutien d'un consultant et du représentant du Développement européen.

\section{Document 3}

L'enquête récente du CILT (1994) sur l'EPLV dans les écoles primaires d'Angleterre, du pays de Galles, et d'Irlande du Nord, n'a généré qu'un nombre restreint de réponses : $40 \%$ des $L E A$. Sur les cinquante-trois $L E A$ qui ont répondu à l'enquête, quarante pratiquent un enseignement des langues dans leurs écoles primaires.

$93 \%$ des écoles enseignent le français (presque 1800 écoles), un peu plus de $3 \%$ l'allemand ou l'italien (environ soixante écoles pour chaque langue), environ trentecinq écoles enseignent l'espagnol. Un petit nombre d'écoles enseigne des langues asiatiques. Cette enquête ne brosse pas un tableau complet de la situation, car de nombreuses écoles privées enseignent depuis longtemps le français dès l'âge de huit ans. Nous ne disposons pas de statistiques concernant ces écoles. Elles représentent environ $10 \%$ des élèves en Angleterre et au pays de Galles.

\section{Document 4}

Le Professeur Hawkins définit quatre grands thèmes et propose des tâches et des activités variées. Les thèmes qu'il propose recouvrent :

Thème 1 - Les formes de langage

Comment communiquons-nous sans langage?

Quelles sont les possibilités/limites des langages des signes?

Comment les oiseaux/animaux communiquent-ils?

Les codes et les codes secrets, les acronymes...

Les types de langage : plus de cent cinquante langues parlées au Royaume-Uni ; les

langues européennes, les langues du monde entier, les familles de langues; les

dialectes, les accents, les registres de langue ; les versions nombreuses et variées de l'anglais (anglais, irlandais, écossais, américain, antillais, australien, africain...) ;

l'appropriation du langage : argot, jargon, mots à la mode.

Langue parlée et écrite : en quoi diffèrent-elles ? les problèmes d'apprentissage de la lecture.

Les formes d'écriture : pictogramme, hiéroglyphe, syllabe, alphabet, phonème.

Thème 2 - La structure du langage

Qu'est-ce qu'une syllabe, une voyelle, une consonne, un phonème, un morphème? Combien de mots connaissent la plupart des locuteurs ? Connaissons-nous tous les mêmes mots? 
Les langues possèdent-elles toutes le même nombre de mots? Pourquoi n'est-ce pas le cas? Que signifie connaître un mot?

Qui décide de la signification d'un mot?

Est-ce la même chose en France, en Espagne, en Allemagne?

Comment l'ordre des mots est-il porteur de sens?

Comment se présente l'ordre des mots anglais par rapport à celui d'autres langues?

Comment le sens est-il modifié par des changements de son, d'orthographe,

d'intonation?

Comment le sens est-il modifié par l'intonation, l'accentuation?

Comment la grammaire exprime-t-elle des données temporelles, spatiales, une

séquence d'événements?

Qu'est-ce qu'une règle de grammaire?

Thème 3 - L'utilisation du langage

Comment les sons sont-ils produits par les organes vocaux?

Quels sons de l'anglais n'apparaissent pas en espagnol, en français, en allemand et vice versa?

Quels sons apparaissent dans les langues parlées par les élèves immigrés mais pas en anglais?

Comparer les différences entre langue parlée et langue écrite :

Qu'est-ce qu'une langue de « mauvaise qualité »?

Qu'est-ce qu'une mauvaise utilisation du langage?

Comment les idées sont-elles liées par le langage?

Qu'est-ce qu'une proposition, une phrase, un paragraphe?

Que sont les devinettes et les jeux de mots, les synonymes, les homonymes?

Pourquoi les devinettes et les jeux de mots sont-ils intraduisibles dans d'autres

langues?

En quoi les registres de langue diffèrent-ils?

En quoi les dialectes diffèrent-ils?

Pourquoi nous moquons-nous des dialectes des autres?

Comment les différences de classes sociales sont-elles reflétées par le langage?

Comment les langues évoluent-elles?

Pourquoi une langue emprunte-t-elle certains mots à d'autres langues?

Thème 4 - L'acquisition du langage (L1 et L2)

Comment les bébés apprennent-ils à parler?

Quand prononcent-ils leurs premiers mots?

De quel genre de mots s'agit-il?

Que comprennent les bébés avant de commencer à parler ?

Quand le discours d'un bébé contient-il pour la première fois des schémas

grammaticaux?

Comment se développe le vocabulaire de la L1?

En quoi est-ce différent pour la L2?

En quoi le dialogue avec les adultes est-il important pour de jeunes enfants?

Quelles sont les principales différences entre l'apprentissage d'une L1 et celui d'une

L2?

Comment pouvons-nous aider les élèves à apprendre la L2 ?

Qu'est-ce qui rend le français, l'espagnol, l'allemand difficiles à apprendre? 
Quels problèmes apparaissent lorsqu'on apprend à lire une L2 ?

Les genres en français, en espagnol, en allemand.

Autres différences entre l'anglais et d'autres langues européennes.

Les idées et les principes sur lesquels se base ce programme ont été publiés par Eric

Hawkins dans son ouvrage intitulé Awareness of Language : An Introduction (Cambridge

University Press, 1984). Le livre était accompagné d'une excellente collection de

fascicules traitant des mêmes thèmes, qui proposent aux enfants des tâches

intéressantes destinées à développer leur recherche et leur réflexion sur le langage et son mode d'utilisation.

\section{NOTES}

1. Burstall et al., (1974).

2. Un bref descriptif de ce projet et de ses caractéristiques est inclus dans le document 1.

3. J'en ai fait un rapide descriptif dans le document 2.

4. D'après un article de Keith Sharpe, in ALL LLJ, $\mathrm{n}^{\circ}$ 6, sept. 92.

5. Publiés chez Didier/Hatier en 1994.

6. SOED, Modern Languages in the Primary School: Competences, 1994.

7. Un résumé de ces propositions se trouve dans le document 4.

8. Édité par CLÉ en 1994.

9. N. D. T.: ces supports sont appelés flashcards. Il s'agit de cartes où figurent des mots, des nombres ou des dessins que l'enseignant montre un court instant à la classe.

10. Publié trois fois par an depuis 1992 par le Verlag Dùrr et Kessler et le Goethe Institut.

11. Ces cours sont disponibles sur trois cassettes vidéo produites par SCOPE Picture Productions Ltd, pour le compte du SOED d'Écosse.

12. Drop in ; se rendre quelque part ou chez quelqu'un de façon imprévue.

\section{RÉSUMÉS}

Après un bref historique sur l'apprentissage précoce des langues dans les écoles primaires britanniques, une réflexion est menée sur les objectifs que cet enseignement doit atteindre aujourd'hui, malgré des difficultés réelles concernant la qualification des enseignants, la langue à enseigner et la didactique à employer.

\section{INDEX}

Mots-clés : école primaire, enseignement des langues, langues étrangères, enseignement précoce des langues vivantes

Index géographique : Grande-Bretagne, Royaume-Uni 


\section{AUTEURS}

\section{PETER SATCHWELL}

Professeur docteur, université de Sussex, Brighton, Grande-Bretagne. 